\title{
Saharan Sand and Dust Storms and Neonatal Mortality: Evidence from Burkina Faso
}

\begin{abstract}
A B S T R A C T
West African populations are exposed to the longest and harshest dust storms on the planet, the Saharan sand and dust storms (SDS). Nonetheless, little is known about the effects of the severe storms on early-life health in West Africa. This study investigated the association of the risk of neonatal mortality, an indicator of the population's early-life health, with potential prenatal and neonatal exposure to the Saharan SDS. Data on 30,552 under-five children from Burkina Faso's 1993, 2003, and 2010 demographic and health surveys were matched to the particulate matters (PM) and terrestrial air temperature and precipitation forecasts. Exposure to dust events was measured by the number of days with average PM10 and PM2.5 concentrations above a series of threshold. Intensitydependent patterns of associations between neonatal mortality and both prenatal and birth month exposure to dust events were identified. There was no association if average daily PM10 and PM2.5 levels were less than 60 and $30 \mu \mathrm{g} / \mathrm{m}^{3}$, respectively. However, strong associations, which increase almost linearly with the intensity of exposure, were identified when daily PM10 and PM2.5 levels ranged between 70-150 and 40-70 $\mu \mathrm{g} / \mathrm{m}^{3}$, respectively. At the higher PM levels, the association for the gestation period decreased, but that for the birth month remained mostly unresponsive to changes in the PM levels. Larger associations were identified when siblings were compared.
\end{abstract}

Keywords:

Sahran sand and dust storms; PM10; PM2.5, Neonatal mortality; NMMB/BSC Dust model; Burkina Faso 


\section{Introduction}

Every year from around late November to mid-March, the dry northeasterly trade wind blows over the Sahara Desert and carries millions of tons of sand and dust over West African countries and creates the longest and harshest sand and dust storms (SDS) on the planet. For days, the dust concentration in some areas exceeds several multiples of the maximum levels recommended by the World Health Organization (WHO 2006; Goudie 2014). The SDS elevate the concentration of particulate matters (PM) to extreme levels and can severely damage human health, especially in early life.

Exposure to PM can cause neonatal mortality either through poor birth outcomes because of mother's exposure (Dadvand et al. 2013; Ritz et al. 2000; Šrám et al. 2005) or through increased the risks of respiratory and cardiovascular disorders in neonates (Al-Hamoud et al. 2018; Šrám et al. 2005). Potential biological mechanisms of the effect of exposure to PM on birth outcomes are elevated maternal vulnerability to infections, increased blood pressure and heart rate, and disturbances of the pituitaryadrenocortical-placental system (Dadvand et al. 2013; Ritz et al. 2000; Šrám et al. 2005). The empirical literature that investigates the effect is rapidly developing. Studying substantially milder conditions than what West African populations experience, researchers have found significant adverse effects of PM on children. For example, significant associations of asthma, allergies, and respiratory infections in children with exposure to PM2.5 are documented (Gehring et al. 2010 and MacIntyre et al. 2011). Also, significant associations between exposure to PM2.5 and PM10 and infant bronchiolitis are reported (Nenna et al. 2017). Moreover, a host of studies-extensively reviewed by Altindag et al. (2017), and Li et al. (2017)—show significant associations between exposure to PM and the key determinants of neonatal mortality, namely, the risks of low birth weight, preterm birth, and being small for gestational age.

The PM literature is focused on developed nations. Little is known about the effects of exposure to PM on early-life health outcomes in less-developed countries, particularly in West African nations that regularly experience extreme levels of exposure to dust PM. Studies that examine the early-life health effects of exposure to Saharan SDS are very recent (Foreman 2018; Goyal et al. 2019; Heft-Neal et al. 2018; Heft-Neal et al. 2019). They use reanalysis PM2.5 data and pool demographic and health survey data from numerous countries to examine child mortality. They generally find that an increase in prenatal and post-birth exposure to PM2.5 is associated with an increase in child mortality.

This study investigates the effect of exposure to Saharan SDS on neonatal mortality in Burkina Faso. It differentiates from the others in its focus on (1) accounting for the 
severity of SDS, (2) one country, Burkina Faso, hence, avoids the problem of unobserved heterogeneities across countries, (3) neonates, who are the most vulnerable children; and (4) both PM2.5 and PM10.

\section{Methods}

\subsection{Data}

This study used daily concentrations of particulate matters (PM2.5 and PM10) from the forecasts of the Multiscale Online Nonhydrostatic AtmospheRe CHemistry model, the NMMB-MONARCH (also known as NMMB/BSC-Dust). The mineral dust module of NMMB/BSC-Dust is the reference model within the World Meteorological Organization's (WMO) Regional Specialized Meteorological Center with activity specialization on Atmospheric Sand and Dust Forecast. The NMMB/BSC-Dust model covers Northern Africa, the Middle East, and Europe from 1979 to 2010. The spatial resolution of the model is $0.5^{\circ} \times 0.5^{\circ}$. The model is described, and its evaluations are reviewed in the Methods Appendix. Currently, the data between the mid-1994 and 1997 contains incorrect calculations and were not used in this study.

This study's weather condition data were Terrestrial Air 'Temperature and Precipitation: 1900-2010 Gridded Monthly Time Series, Version 3.01 (Matsuura and Willmott 2012). This dataset provides worldwide (terrestrial) monthly average temperature and precipitation at the spatial resolution of $0.5^{\circ} \times 0.5^{\circ}$. The data is used and discussed in other research (Dell et al. 2012; Tanahte et al. 2012; Schneider et al. 2014).

Burkina Faso children's health microdata came from demographic and health surveys (DHS), provided by the DHS Program. This study focused on Burkina Faso because the country is among those whose population is exposed to the most extreme Saharan SDS according to the data from NMMB/BSC Dust model's forecast. In addition, neonatal mortality in the country is one of the highest in the world (GBD 2016 Risk Factors Collaborators 2017). Moreover, Burkinabe women are as exposed to air pollution as men because of the country's predominantly agricultural economy and the high rate of women's labor force participation (World Bank 2017). Nonetheless, to our knowledge, there is no examination of the potential health effects of the Saharan SDS in Burkina Faso. From a practical point of view, Burkina Faso had the most number of DHS datasets that provide household geographical information and overlap with the coverage of the NMMB/BSC Dust data (2/1979 to 3/2010, missing 1994-1997). Specifically, three Burkina Faso surveys were selected for this analysis. They were conducted in 1993, 2003, and 2010, providing information on 5,828, 10,575, and 14,149 
children. In the Methods Appendix, The DHS Program's surveys' design and this study's data selection procedure are described.

\subsection{Measures of exposure to dust events}

The above three datasets were matched using geographical information. Then, the fully matched dataset was used to find temperature, precipitation, and PM levels during children's prenatal and neonatal. The birth month (i.e., the calendar month in which a child was born) was considered as the neonatal period, and the nine months before the birth month was considered as the prenatal period. In the Methods Appendix, the reason for making the assumptions and their potential implications are discussed. Also, the details of processing each dataset and their matching are described.

The presumed prenatal period was divided into three trimesters. Then, in each period, the number of days with PM10 concentrations beyond $10,20, \ldots, 500 \mu \mathrm{g} / \mathrm{m}^{3}$ as well as the number of days with PM2.5 concentrations beyond $10,20, \ldots, 200 \mu \mathrm{g} / \mathrm{m}^{3}$ were calculated. Each PM threshold level indicates a dust event. Formally, Four different sets of exposure measures were defined:

PM10 measures:

$\{$ OverxPM10bmon, OverxPM10gest\}

$\{$ OverxPM10bmon, OverxPM10t1, OverxPM10t2, OverxPM10t3\}

where $\quad \boldsymbol{x}=10,20, \ldots, 500 \mu \mathrm{g} / \mathrm{m}^{3}$

PM2.5 measures:

$\{$ Over $\mathbf{x} P M 2.5$ bmon, Over $\mathbf{x} P M 2.5$ gest $\}$

$\{$ Over $\mathbf{x} P M 2.5$ bmon, OverxPM2.5t1, Over $\mathbf{x} P M 2.5 t 2$, Over $\mathbf{x} P M 2.5 t 3\}$

where $\quad \boldsymbol{x}=10,20, \ldots, 200 \mu \mathrm{g} / \mathrm{m}^{3}$

where OverxPM10 $\boldsymbol{p}$ is the number of days in period $\boldsymbol{p}$ with PM10 beyond $\boldsymbol{x} \mu \mathrm{g} / \mathrm{m}^{3}$. The indicator of period, $p$, is either bmon, gest, $t 1, t 2$, or $t 3$, indicating the birth month, the entirety of gestation, trimester 1 , trimester 2 , and trimester 3 , respectively. For example, 
Over200PM10gest is the number of days in the presumed gestation with PM10 level beyond $200 \mu \mathrm{g} / \mathrm{m}^{3}$.

\subsection{Empirical models}

The intensity of dust concentration varied considerably during the year and across locations in Burkina Faso. At the peak of the storms, according to the NMMB/BSC Dust models' forecasts, the difference between PM10 concentrations in high and low latitude grids could be as high as $500 \mu \mathrm{g} / \mathrm{m}^{3}$; the difference for PM2.5 could be as high as $100 \mu \mathrm{g} / \mathrm{m}^{3}$. Since the exposure measures vary across both time and place of birth, the difference-in-differences approach can be used to control for all confounders that are constant over time or place of birth (A child's place of birth and current residence were assumed the same in this study because the place of birth is not reported in the data). This allows for identifying the effect of exposure to dust events on neonatal mortality. Model 1 shows our basic difference-in-differences specification:

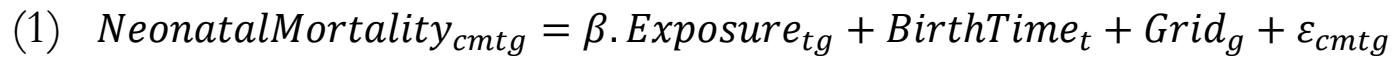

in which NeonatalMortality ${ }_{c m t g}$ is a dummy variable equal to one if child $c$, who was born to mother $m$ at time $t$ in grid (location) $g$, was deceased as a neonate (or in the month of birth) and zero otherwise. Time $t$ represents a season in a specific year (for example, spring of 1998.) The variable BirthTime $_{t}$ is the year-specific season of birth fixed-effect (FE). It captures all confounders that vary from one season to another but are the same all over Burkina Faso. This includes all the seasonal factors, as well as climatic, environmental, social, economic, and political conditions that were experienced by everyone in Burkina Faso in a specific season in a particular year. For example, this variable can capture a country-wide drought in any season during the period of study. The variable Grid $_{g}$ is the FE for the grid $g$ that contains the place of birth. It controls for all confounders that vary from one grid (of birth) to another but do not change over our period of study. This includes grid-specific features, such as the geographic, climatic, environmental, cultural, economic, social, and political characteristics of each grid that do not change over time. For example, the fact that some regions are consistently less-developed than others over time is captured by this FE. 
The variable Exposure $t g$ is a set of exposure measures to dust events that were presented in the previous section. Since it varies over both time and grid (of birth), it acts like the interaction of BirthTime and Grid in the difference-in-differences specification. Thus, its coefficient, $\beta$, depicts the effect of an extra day of exposure to over $\boldsymbol{x} \mu \mathrm{g} / \mathrm{m}^{3}$ PM10 or PM2.5 during the birth season or a gestation period on neonatal mortality, the dependent variable.

By controlling for BirthTime t $_{t}$ and Grid $_{g}$, the difference-in-differences approach accounts for all observable and unobservable confounders that vary by season but are constant across grids, or differ from one grid to another but are constant over time. Hence, it provides a robust estimate of causal effects, as long as there are no confounders that vary across both season and grid of birth. Nevertheless, there could still be confounders that vary across both season and grid of birth or confounders that vary by child and bias the estimates. The most important of these confounders include temperature and precipitation. Therefore, in Model 2, Tempreture $_{t g}$ and Precipitation $_{t g}$ are controlled. They, respectively, are the average temperature (in ${ }^{\circ} \mathrm{C}$ ) and the average precipitation (in $\mathrm{mm}$ ) in grid $g$ in periods for which dust events are considered.

To increase the precision of the estimates, one can add variables at the household and individual levels that could be related to neonatal mortality. At the individual level, the most important set of variables is the mother's characteristics. Mother $_{c m g}$ in Model 2 represents this set and includes the mother's age, age squared, age cubic, religion, ethnicity, education, number of children ever born, number of deceased children, employment, marital status, and relationship to the household head. Another set of individual characteristics relates to the child. $O w n_{c t g}$ represents this set and contains the neonate's sex and birth order and a twin indicator. Household ${ }_{c m g}$ in Model 2 represents household-level variables. In this analysis, they are an urban/rural indicator, number of members, number of under-five children, number of women in the household, mother's partner's education, and a set of asset-ownership variables (access to electricity, TV and radio ownership, floor type, toilet type, and the source of drinking water.)

In addition, any possible differential seasonal effects in location characteristics (for example, because some locations may experience less economic activity during certain seasons than other locations) were controlled by the interaction of birth season (not year-specific) and Grid $_{g}$, the variable $L_{s g}$. 


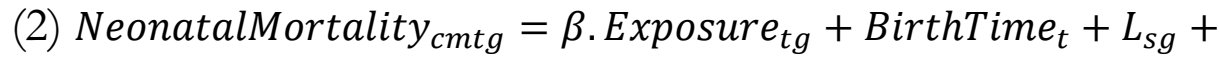

$$
\begin{aligned}
& \delta . \text { Tempreture }_{t g}+\pi . \text { Precipitation }_{t g}+ \\
& \mu . \text { Mother }_{c m g}+\omega . \text { Own }_{c g}+\theta . \text { Household }_{c m g}+ \\
& \varepsilon_{c m t g}
\end{aligned}
$$

There could still be many (time-constant) unobservable factors, particularly pertaining to the mother that can affect neonatal mortality. One example of such unobservable characteristics is the mother's preferences for investment in health. Exclusion of such unobservable factors does not bias the results in Model 2 as long as they are timeinvariant, because they would be captured by the grid FEs. Nonetheless, to increase precision, one may want to include mother's FE rather than grid FE to control for mother's time-invariant unobservable factors. Model 3 depicts such a specification:

$$
\begin{aligned}
\text { (3) } \text { NeonatalMortality }_{c m t g}= & \beta . \text { Exposure }_{t g}+\text { BirthTime }_{t}+\varphi_{m}+L_{s g}+ \\
& \delta . \text { Tempreture }_{t g}+\pi . \text { Precipitation }_{t g}+ \\
& \omega . \text { Own }_{c m g}+\varepsilon_{c m t g}
\end{aligned}
$$

in which $\varphi_{m}$ is the mother's FE. By including mother's FE, grid FE (i.e., Grid ${ }_{g}$ ) drops out on the model. The FE model added an extra level of comparison to the analysis: it compared children of the same mother. Since living children of different mothers were at different ages, children's age in months was also included in the vector $O w n_{c m g}$. The vectors Mother $_{\text {cmg }}$ and Household ${ }_{c m g}$ were eliminated because of the FE structure. The FE model uses mothers with at least one deceased child and one living child to identify the effect of dust events. Nonetheless, all mothers, even those with only one child, were included in the FE estimation sample to have the same sample composition in all three models.

Models 2 and 3 are not completely immune to the influence of confounding factors. For example, parents may decide to migrate to areas less prone to SDS after observing the effect of exposure to SDS on their first child's health. Also, the family may migrate between the birth of their first and second children for economic reasons. Such possibilities cannot be directly checked in this analysis since families were not followed over time in the DHS data. The impact of not accounting for such cases, however, may 
be small since Burkina Faso's decennial censuses show that the percentage of the population in its 45 provinces remained largely unchanged from 1985 to 2017 (http://www.insd.bf/n/). Moreover, the FE estimates should be interpreted cautiously because of their limited statistical power: the total number of deceased neonates in our FE regressions was 989; among them, about 264 were their mothers' only reported birth.

Since PM10 and PM2.5 concentration levels in Burkina Faso were strongly correlated, single pollutant-i.e., PM10- and PM2.5-specific-models were estimated. These models were estimated using linear probability rather than logit, because, in differencein-differences models, the coefficient of interest is the coefficient of the interaction term. Using linear probability, the coefficient of the interaction term (Exposure ${ }_{t g}$ ) is readily interpretable as the increase in the probability of neonatal mortality due to an increase in exposure. If the logit model is used instead, the coefficient of the interaction term shows the ratio of odds ratios between the treated and untreated and has a complicated and confusing interpretation. It is, therefore, uncommon to use logit to estimate difference-in-differences models. For comparison, however, logit regressions were estimated, and their average marginal effects were reported in the Supplementary Tables and Figures. In all estimations, standard errors were clustered at urban-region units within a province-clustering at the geographical unit controls for autocorrelation in data series (Bertrand et al. 2004). For brevity, Models 2 and 3 are referred to as LPM (Linear probability Model) and FE hereafter.

\section{Results}

\subsection{The PM concentration in Burkina Faso}

The WHO air quality guidelines suggest below 50 and $25 \mu \mathrm{g} / \mathrm{m}^{3}$ as targets for 24-hour PM10 and PM2.5 concentration levels. The guidelines also recommend three interim targets levels: 150,100 , and $75 \mu \mathrm{g} / \mathrm{m}^{3}$ for PM10 and 75, 50, and $37.5 \mu \mathrm{g} / \mathrm{m}^{3}$ for PM2.5 (WHO 2006). The WHO's review of epidemiological and clinical studies showed that at interim target-1 (i.e., 150 for PM10 and $75 \mu \mathrm{g} / \mathrm{m}^{3}$ for PM2.5), daily dust-related mortality was about 5\% greater than that at the target level; at interim target- 2 and target-3, it was about $2.5 \%$ and $1.25 \%$ greater than that at the target level (WHO 2006).

The WHO risk assessments were based on easily observed, short-term exposure to elevated levels of PM, whereas long-term risks are much higher (WHO 2013). In Burkina Faso, both the level and the length of exposure to dust are extreme. Supplementary Figures 1-3 show the time-series of daily PM concentration levels for 
two selected Burkina Faso locations with usually the highest and the lowest dust concentration levels in the country. The location with the highest concentration is near the city of Kantchari at the border with Niger in the east. The location with the lowest concentration is near the city of Mangodara at the border with Ivory Coast in the south. Dust concentration in other grids usually falls between the levels in these two locations. The trends show that in most days during the dust season (the late November to the mid-March), PM10 and PM2.5 concentrations were far above even the WHO interim target-1. They surpassed 1,000 and $200 \mu \mathrm{g} / \mathrm{m}^{3}$ frequently and even reached as high as 2,000 and $500 \mu \mathrm{g} / \mathrm{m}^{3}$.

Table 1a shows the mean and median values of average PM10 by birth month. In winter, which largely overlaps with the dust season, even the monthly averages of daily PM10 concentrations went well above the WHO maximum interim target. Monthly average exposure levels gradually decrease for those born in spring and summer, reaching their lowest levels in August and September. They slowly rise during autumn. The number of days in which PM10 levels passed the $150 \mu \mathrm{g} / \mathrm{m}^{3}$ threshold was also significantly larger in winter. The mean and median numbers of such days were 14 in January and remained in two digits in February and March. The average mean and median for other months were 3.4 and 2.4 days, respectively. Table $1 \mathrm{~b}$ presents a similar pattern of the birth month PM2.5 concentration. If the birth month is January, February, or March, the mean and median values of average PM2.5 went well above 50 $\mu \mathrm{g} / \mathrm{m}^{3}$, the WHO medium interim target. Also, in many days of the winter months, PM2.5 level passed $50 \mu \mathrm{g} / \mathrm{m}^{3}$ threshold: mean numbers of such days were 14,10 , and 15 in January, February, and March, respectively.

\subsection{Uncontrolled analysis of observables}

Table 2 compares the number and percentage of births and deaths by birth season. It also compares children born in different seasons in terms of an array of characteristics of the weather and their mothers, fathers, and households. In winter, during which dust concentrations are usually the highest in Burkina Faso (Table 1), the percentage of births was slightly less than that in other seasons, especially in the north and south regions and in the capital city. Nonetheless, the rates of deaths on the first day and the first month after birth were very close across birth seasons.

There was high birth-season heterogeneity in precipitation rate and some heterogeneity in temperature, region, mother's and mother's partner's education level, mother's religion and ethnicity, and household's water source. For example, a relatively higher 
proportion of children in the sample were born in winter in the western and eastern regions. Also, when the mother or her partner was more educated (i.e., completed primary or some secondary school education), then birth in winter was slightly less likely.

Although not presented in Table 2, mothers were compared in terms of their awareness of the ovulatory cycles and access to contraceptive methods. Less than $20 \%$ of mothers were aware of the ovulatory cycle, and more than $80 \%$ of them had no access to contraceptive methods. This information may indicate a limited ability to plan conception. This and similar confounders can be captured by the mother's FE in Model 2.

\subsection{The models' estimations}

Figures 1 and 3 show the results based on Model 2 (LPM model) for PM10 and PM2.5, respectively. Figures 2 and 4 report the estimates based on Model 3 (FE model) for PM10 and PM2.5, respectively. The horizontal axes in all these figures show various thresholds for PM10 and PM2.5. The vertical axes depict the number of neonatal deaths out of 100,000 births for every extra day in which PM levels surpassed the thresholds on the horizontal axes. This is the coefficient $\beta$ in Models $1-3$. The vertical lines in these figures show the $95 \%$ confidence intervals for the estimated effects. The results for both LPM and FE models show that the effect of PM on neonatal mortality was not statistically significant at low- to mid-level concentration levels-namely, at up to about $60 \mu \mathrm{g} / \mathrm{m}^{3}$ for PM10 and $30 \mu \mathrm{g} / \mathrm{m}^{3}$ for PM2.5. The effect, however, becomes statistically significant afterward and almost linearly increasing until about 150 and 70 $\mu \mathrm{g} / \mathrm{m}^{3}$ thresholds for PM10 and PM2.5, respectively. For example, according to Figure 1, an extra day with PM10 levels above $170 \mu \mathrm{g} / \mathrm{m}^{3}$ in the month of birth increases the number of neonatal deaths per 100,000 births by 114. For higher thresholds, the size of the effect of an extra day of exposure during the birth month stays roughly constant. It becomes statistically indistinguishable from zero for pre-birth exposure. Since the results from models with exposure measures for the entirety and trimesters of gestation were very similar, the trimesters' results are presented among the Supplementary Tables and Figures.

There could be behavioral, socioeconomic, or physiochemical reasons for the fact that the rate of increase in neonatal mortality slowed down as dust concentration intensified. For example, when the dust particles' concentrations are clearly high, common sense encourages pregnant women or new mothers to take extra care and protect themselves 
and their newborns by minimizing their exposure to dust. Also, working pregnant women may leave their place of residence less frequently because of the possible slowdown of economic activities during severe SDS. In addition, the particles may form co-aggregates and sediment in the ground surface faster and hence, penetrate the respiratory system less. When the concentration is not very high, the particles-which are natural colloidal or even nanosized mineral dust—remain suspended in the air, apart from each other and might repel each other due to having a similar surface charge (Gao and Zhang 2002; Xie et al. 2010).

In terms of the effect size, estimations from Model 2 show that one extra day of gestational and birth month exposure to an over $80 \mu \mathrm{g} / \mathrm{m}^{3}$ PM10 dust event was associated with an average 13 (95\% Confidence Interval: -1-27) and 50 (95\% CI: 1881 in 100,000 increase in neonatal mortality, respectively. The magnitude of the association reached 39 (95\% CI: -4-81) and 99 (95\% CI: 41-57) for one extra day of gestational and birth month exposure to an over $150 \mu \mathrm{g} / \mathrm{m}^{3}$ PM10 dust event, respectively. The size of the association even increased to 139 (95\% CI: 98-180) for one extra day of birth month exposure to an over $390 \mu \mathrm{g} / \mathrm{m}^{3}$ PM10 dust event (Figure 1). Higher associations were found from the FE model. The size of the corresponding FE associations were 57 (95\% CI: 5-110) and 115 (95\% CI: 21-209) for gestational and birth month exposure to an over $80 \mu \mathrm{g} / \mathrm{m}^{3}$ PM10 dust event, respectively. They were 97 (95\% CI: 25-168) and 182 (95\% CI: 63-301) for gestational and birth month exposure to an over $150 \mu \mathrm{g} / \mathrm{m}^{3}$ PM10 dust event, respectively (Figure 2). The pattern and size of the associations from trimester estimations were consistent with those observed from the entirety of gestation estimations (Supplementary Figures 5 and 6). Noticeably, more or less, the same trend in the associations was measured for exposure during all three trimesters. Moreover, the results from using an alternative estimation method of Model 2, Logit regression, resulted in the average marginal effects that were very close to the marginal effects founds from the LPM (Supplementary Table 1).

In addition, Model 2 estimates for PM2.5 show that one extra day of gestational and birth month exposure to an over $40 \mu \mathrm{g} / \mathrm{m}^{3} \mathrm{PM} 2.5$ dust event was associated with an average 15 (95\% CI: -1-31) and 59 (95\% CI: 26-93) in 100,000 increase in neonatal mortality, respectively. The magnitude of the association reached 54 (95\% CI: 1-108) and 125 (95\% CI: 71-179) for one extra day of gestational and birth month exposure to an over $70 \mu \mathrm{g} / \mathrm{m}^{3}$ PM2.5 dust event, respectively. The size of the association reached 205 (95\% CI: 58-352) for one extra day of birth month exposure to an over $190 \mu \mathrm{g} / \mathrm{m}^{3}$ PM2.5 dust event (Figure 3). Similar to the PM10 examination, higher associations were found from the FE model. The size of the corresponding FE associations were 43 (95\% CI: -7-92) and 135 (95\% CI: 41-229) for gestational and birth month exposure to an 
over $40 \mu \mathrm{g} / \mathrm{m}^{3}$ PM2.5 dust event, respectively. They were 78 (95\% CI: 7-149) and 179 (95\% CI: 66-292) for gestational and birth month exposure to an over $60 \mu \mathrm{g} / \mathrm{m}^{3} \mathrm{PM} 2.5$ dust event, respectively (Figure 4). These results were in line with those found from trimester and Logit model estimations (Supplementary Figures 7 and 8; Supplementary Table 1).

The average PM concentration levels during gestation and birth month were also calculated and used as measures of exposure in the statistical models. Regardless of the statistical model and the PM size, an increase in the average dust concentration during the gestation period had no statistically significant association with neonatal mortality. The increase in the average PM level during the birth month was strongly associated with neonatal mortality in all settings (Supplementary Table 2). According to the LPM with the entirety of gestation exposure measure, a $10 \mu \mathrm{g} / \mathrm{m}^{3}$ increase in PM10 concentration during the birth month was associated with 57 (95\% CI: 27-86) in 100,000 more neonatal deaths. The same with the trimester exposure measures was 61 (95\% CI: 26-95). The same size increase in PM2.5 level was associated with a much greater neonatal mortality, 193 (95\% CI: 86-300) and 203 (95\% CI: 77-329) in 100,000 from the LPM with the entirety and trimesters of gestation exposure measures, respectively. For both PM10 and PM2.5, the FE estimates were about double the LPM estimates: 103 (95\% CI: 18-188) and 378 (95\% CI: 90-666) in 100,000 for PM10 and PM2.5, respectively, when the entirety of gestation exposure measure was used. Average exposure measures, however, mask important variation in PM concentration during gestation and birth month, as they do not account for the severity of exposure to dust events. Hence, they do not offer much insight into the effects of exposure to extreme events. In the analysis sample, $40 \%(35 \%), 15 \%(12 \%)$, and $8 \%(8 \%)$ of all children were exposed to over 100 (50), 200 (80), and 300 (100) $\mu \mathrm{g} / \mathrm{m}^{3}$ PM10 (PM2.5) levels for at least 10 days in their birth month.

To put the estimates of the potential effect of exposure to dust events in perspective, the association of neonatal mortality with a "10 day" exposure to a dust event was compared to that with other statistically significant factors. Winter-born children were exposed to at least 10 days with PM10 beyond $150 \mu \mathrm{g} / \mathrm{m}^{3}$ and at least 10 days with PM2.5 beyond $50 \mu \mathrm{g} / \mathrm{m}^{3}$, on average (Table 1). If the association of neonatal mortality with 10 days of exposure to a dust event can be assumed 10 times the association of neonatal mortality with one day of exposure to the event, then the association of neonatal mortality with a 10-day exposure to a dust event with PM10 (PM2.5) level beyond $150(50) \mu \mathrm{g} / \mathrm{m}^{3}$ was 990 (770) in 100,000 (Figures 1 and 3). Corresponding FE estimates of the associations were about two times greater. 
Among the other risk factors, twin birth had a notable association with neonatal death: it was associated with 8,400 (95\% CI: 6,028-10,772) more neonatal deaths in 100,000 (The number of twins in the sample is 966 , about $3 \%$ of all children). Another factor that had a large association with the risk of neonatal mortality was birth order: a oneunit increase in birth order was associated with 7,190 (95\% CI: 6,294-8,086) in 100,000 decreases in neonatal mortality. This finding can be related to the mother's age. Mothers' age in the analysis sample ranges from 16 to 49 years; about $60 \%$ are 30 years old or younger. As mothers become older and more experienced, the chance of neonatal death decreases. Accordingly, a one year increase in mother's age was associated with 1,310 (95\% CI: 630-2,010) in 100,000 decreases in neonatal mortality. Also, presence of one more woman in the household was associated with 1,520 (95\% CI: $1,097-1,951)$ in 100,000 decreases in neonatal death, reflecting the effect of experience sharing and childcare assistance in a household (The average number of women in a household in the sample is 2.0.). If the mother was from Lobi ethnic-group, then her neonate had a higher chance of survival by 894 (95\% CI: 484-1,484) in 100,000 than when she was from Bobo ethnic-group (Supplementary Tables 3 and 5).

Among the indicators of wealth, floor type had a significant association with neonatal mortality: the chance of neonatal death was by 820 (95\% CI: 97-1,543) in 100,000 higher in households in which the floor of the place of residence was simply naturewhich is the case for about $61 \%$ of the households in the sample-than in household with the floor type of asphalt, cement, parquet, ceramic, or carpet. Also, the chance of neonatal death was higher in male children by 784 (95\% CI: 306-1,262) in 100,000 (Supplementary Tables 3 and 5). About 51\% of the children in the analysis sample are male.

The associations of neonatal mortality with a twin birth, birth order, mother's age, presence of other women in the household, floor type, and child's sex were almost identical in PM10- and PM2.5-specific regressions. When mother's fixed-effect was included, the even greater associations were found: twin birth, being a male child, and being the first child were respectively associated with 14,900 (95\% CI: 10,098-19,702), 11,000 (95\% CI: 8,864-13,136), and 1,200 (95\% CI: 359-2,041) in 100,000 more neonatal deaths. These results suggest that, except for twin birth and birth order, the risk of potential exposure to particulate matters lifted by the Saharan SDS was comparable to the socioeconomics and biological risks of neonatal mortality. The association of neonatal mortality with the mother's education was not statistically significant, a seemingly surprising result. It, however, was the consequence of limited variation in mother's education in the sample, more than $85 \%$ of them are illiterate, and 
only about 4\% of them have more than primary education (Supplementary Tables 4 and 6).

The relationship between neonatal mortality and weather characteristics was not consistent. The temperature and precipitation during the gestational period were positively associated with neonatal mortality in the LPM estimates. In the FE model, however, no association was found for temperature, and precipitation's association transferred to the birth period (Supplementary Tables 4-6).

Whether the association of neonatal mortality with exposure to dust events varies by socioeconomic and biological factors was investigated as well. In practice, dummy variables that indicate if a child's birth order is 1 or 2 , if the floor type is nature, and if the child is a male were generated. Then, they interacted with measures of exposure to PM10 and PM2.5 in Models 2 and 3. The exercise was not conducted for twins and mother's age because the numbers of twins and the number of very young (younger than 19) and very old (40 or older) mothers are not large enough to render statistical power to the tests. The results, however, did not show that the association of neonatal mortality with exposure to dust events was stronger among first or second children, in poorer households (measured by floor type), and in male children. These results are consistent with the results of other studies (e.g., Currie et al. 2009 and Heft-Neal ett al. 2018). However, differential effects of exposure to air pollutants by risk factors of pregnancy, such as anemia, hypertension, and eclampsia are documented in other research (e.g., Currie et al. 2009). Information on such complications is not available in the Burkina Faso data.

\section{Discussion}

\subsection{Contents of the Saharan dust}

One key determinant of the effect of PM on health is its size. The effect on birth outcomes of exposure to PM2.5 (or fine particle) is usually greater than that of PM10 (or coarse particle) since the first can reach deeper into the lungs and thus is more dangerous for the human health (EPA 2009). Another factor is the PM's chemical composition, which is different by source (WHO 2013). The main content of deserts' dust is silica (silicon dioxide), a compound of minerals with high quartz contents. Inhaling silica is toxic and causes severe inflammation of lung tissues, silicosis, bronchitis, rheumatoid arthritis, and lung cancer (Middleton 2017). Some deserts' dust can also contain heavy metals and other irritating or toxic minerals such as arsenic, 
polychlorinated biphenyls (PCBs), polycyclic aromatic hydrocarbons (PAHs), and pesticides (Garrison et al. 2006; Soukup et al. 2012). In addition to minerals, desert dust contains microorganisms such as bacteria, viruses, fungi, and pollen spores, which can survive a global scale transport. The microbiological materials can cause or increase the incidence of coccidioidomycosis, meningococcal meningitis, conjunctivitis, and dermatological disorders, in addition to respiratory and cardiovascular diseases (Goudie, 2014; Perez Garcia-Pando et al. 2014a,b).

Research examining the contents of the Saharan dust is scarce. Few small-scale studies show that it contains a series of bacterial and fungal genera (Kellogg et al. 2005), Neisseria meningitis (Thomson et al. 2009), and pesticides and heavy metals (Garrison et al. 2006). Exposure to such contents can potentially lead to disorders, infections, or inflammations in pregnant women and result in adverse pregnancy outcomes (Goldenberg et al. 2008; Purisch et al. 2017; Koullali et al. 2017). To our knowledge, there is no research on the contents of the dust that covers Burkina Faso during the Harmattan season.

\subsection{Implications of the strong correlation between PM10 and PM2.5 levels}

PM10 and PM2.5 levels in Burkina Faso during the period of this study were strongly correlated because of their common source and the small contribution of non-natural sources into the total concentration of PM in the country. To show their almost perfectly parallel trends, their 2004-2009 time-series at the Burkina Faso capital city, Ouagadougou, was graphed (Supplementary Figure 4). Also, the Pearson correlation coefficient for average PM10 and PM2.5 levels during both birth month and the gestation period was about $97 \%$. As a result, common symptoms of perfect collinearity appeared in statistical analyses when both PM10 and PM2.5 exposure measures were included.

The strong correlation between PM10 and PM2.5 measures, however, has important implications for the interpretation of the estimated associations: the measured associations for PM10 can be attributed to PM2.5 and vice versa. Since the association of neonatal mortality with exposure to PM2.5 was noticeably greater than that with exposure to PM10, it can be plausibly deduced that exposure to the Saharan PM2.5 was positively associated with neonatal mortality. Nonetheless, how much of the association was influenced by PM10 or if PM10 had any association with neonatal mortality cannot be known. In general, it may be concluded that the association of neonatal mortality with exposure to PM falls in the range of associations measured for PM10 and PM2.5. 


\subsection{Birth month as the neonatal period}

Because of the day of birth data limitations (explained in the Methods Appendix), the birth month was considered as the neonatal period. In fact, for most children, the neonatal period extends beyond the month of birth. If the birth month does not include all neonatal days, then neonatal mortality can be the result of unaccounted exposure to dust events in the days that fell outside the birth month. Nonetheless, this is not a strong assumption because the majority of neonatal deaths in this analysis had taken place in the first two weeks after birth-in line with what has been documented in other countries (Lawn et al. 2005; Lawn et al. 2010). Specifically, 87\%, 74\%, and $20 \%$ of all neonatal deaths in the study sample happened in the first two weeks, the first week, and the first day after birth, respectively. Therefore, for the majority of children, the birth month encompassed the most crucial days of the neonatal period in terms of the risk of death.

In a robustness test, measures of exposure to dust events during the month after the birth month were included in the models-however, no statistically significant association between neonatal mortality and exposure to dust events during that month was found. Also, the size and pattern of the gestation period and birth month associations remained close to the original ones (Supplementary Figures 9-12). The fact that inclusion of the next month's exposure measures does not affect the pattern of the results reduces the concern that the results are driven by unobserved confounding factors that are correlated with both neonatal mortality and PM concentration.

\subsection{Exposure during gestation}

Since the mother's body blocks air dust pollution from reaching the fetus, neonatal mortality's association with prenatal exposure to dust events is expectedly weaker than its association with postnatal exposure. The finding, however, must not undermine the importance of protecting pregnant women from exposure to SDS because the mother's health condition can still influence a fetus's growth and health even if it does not lead to mortality.

Although the association of neonatal mortality with prenatal exposure to PM might be considered small in comparison to other factors, its association with birth weight and gestational age- key markers of long-term health — can be large, as documented by a body of literature (WHO, 2013). In fact, in Burkina Faso, during 1990 to 2016, 9.5\% to $10.5 \%$ of the burden of diseases were attributable to low birth weight and short gestation (GBD 2016 Risk Factors Collaborators, 2017). 
For two reasons, the DHS data does not allow for measuring the association of birth weight and gestational age with prenatal exposure to dust events. First, no information on the length of gestation is reported in the data. Second, the reported birth weight data are extremely heaped, as they are based on mothers' recall.

\subsection{The potential influence of other air pollutants}

In addition to the PM level, the ambient temperature and humidity change during desert SDS (Johnson et al. 2011; Moreira et al. 2019). Desert SDS can also change ozone $\left(\mathrm{O}_{3}\right)$ and nitrogen dioxide $\left(\mathrm{NO}_{2}\right.$ ) concentration (Johnson et al. 2011; Moreira et al. 2019), although there are studies that show their levels may remain low (Xie et al. 2005). All these factors can independently increase the risk of mortality (Anderson and Bell, 2009; Ito et al. 2005) and hence, modify the measured associations in this analysis. Temperature and humidity are already in this analysis. $\mathrm{O}_{3}$ and $\mathrm{NO}_{2}$, however, may not be significant confounders in this analysis.

$\mathrm{O}_{3}$ and $\mathrm{NO}_{2}$ levels can be elevated during desert SDS for several reasons. One is biomass combustion because of forest fires or by household's use of biomass fuel (Johnson et al. 2011; Moreira et al. 2019). The only available data on exposure to air pollutants in the household is the type of cooking fuel, provided in two of the three Burkina Faso DHS datasets used in this study. According to this variable, 94\% of households used wood as their home cooking fuel. For 97\%, this fuel was either wood or charcoal. This leaves practically no variation across the households in terms of exposure to the air pollutants emitted from home cooking fuel. Hence, the home fuel variable and its interaction with the PM exposure measures were automatically eliminated from the regressions by the statistical software.

$\mathrm{O}_{3}$ and $\mathrm{NO}_{2}$ can also be generated from human economic (e.g., industrial and transportation) activities. The amounts of $\mathrm{O}_{3}$ and $\mathrm{NO}_{2}$ originated from economic activities are not likely to be substantial in West Africa (Heft-Neal et al. 2018). This particularly applies to Burkina Faso, where about $90 \%$ of the population works in agriculture, and the industrial sector is a hundred times smaller than that in Spain, which is partially affected by the Saharan SDS (CIA 2020; Moreira et al. 2019).

\subsection{Comparison to other analyses}

All recent analyses of the relationship between child mortality and the Saharan SDS pooled data from numerous countries. Specifically, Foreman (2018), Goyal et al. (2019), 
Heft-Neal et al. (2018), and Heft-Neal et al. (2019) pooled data from 11, 30, 30, and 43 countries, respectively. All the studies used child health data from the demographic and health surveys of The DHS Program, as did this analysis. This study, however, avoided pooling data from different countries for several important reasons.

First, it introduces significant heterogeneities in the sample. The heterogeneities may not always be observed or controlled by country fixed-effects, especially when they are time-variant. Economic growth is a good example of such confounders. It varies across countries and over time. On the one hand, it affects the expansion (or contraction) rate of industries and transportation facilities, which changes PM exposure. On the other hand, it can affect the development of the healthcare systems, which affects neonatal mortality. As the number of countries increases, the number of potential confounders that vary across both time and space increases, and the likelihood of getting biased estimates rises. Confining the study to a single country significantly reduces the risk of such biases.

Second, the number, size, and quality of surveys from different countries are very different. For example, during 1988-2010, there are 4, 3, 2, 1 survey(s) from Nigeria, Benin, Ivory Coast, and Sierra Leone with the total number of 46.5, 24, 6, and 5.5 thousand under 5-year-old children. There could be political and economic reasons that affect the size and quality of such surveys. If these reasons are correlated with both exposure and mortality and vary across time and space, the results would be biased. For example, imagine the outbreak of a civil war dissuades authorities from collecting DHS in a country. Civil war can increase exposure by destroying physical barriers against dust events (e.g., buildings.) It can also affect neonatal mortality by the destruction of the healthcare infrastructure. Hence, countries would have different sample sizes for reasons that are correlated with both exposure and mortality. The difference in the sample sizes cannot be corrected with weights or any other method. Third, it will not be possible to draw policy for a specific country when results are from a pooled sample.

In addition, this study differs from the other studies in how it is specially designed to account for the severity of dust events. First, the preferred exposure measures in this study are the number of days during which PM levels surpass the thresholds that increase from 10 to 200 and $500 \mu \mathrm{g} / \mathrm{m}^{3}$ for PM2.5 and PM10, respectively. The other studies used the sum or average of the PM during long pre- and post-birth episodes, hence, smoothened the intensity of severe dust events. Second, like Foreman (2018), this study focuses on West Africa, where the SDS are most severe. The other three studies include countries from Southern Africa and Asia as well. 
While this study used the same source for children's information, its source of PM data is different from the others. Foreman (2018) used MERRA-2 aerosol optical depth data, which has a spatial resolution of $0.5^{\circ} \times 0.625^{\circ}$ (Roland et al. 2017). Goyal et al. (2019), Heft-Neal et al. (2018), and Heft-Neal et al. (2019) used the PM2.5 estimates of the Atmospheric Composition Analysis Group at Dalhousie University (van Donkelaar et al. 2016). The spatial resolution of the latter is $0.01^{\circ} \times 0.01^{\circ}$, substantially higher than $0.5^{\circ} \times 0.5^{\circ}$ - the spatial resolution of data from the NMMB/BSC-Dust model, used in this analysis. The NMMB/BSC-Dust data, however, has three advantages over the data from the Atmospheric Composition Analysis Group. First, they provide both PM2.5 and PM10 concentrations and hence, allow for a comparison between PM2.5 and PM10 effects. Second, they cover a more extended period: 1979-2010, versus 1998-2014. Therefore, NMMB/BSC-Dust data can be matched with more DHS datasets. Third, the NMMB/BSC-Dust model is certified by the United Nations (U.N.) and used by the WMO Sand and Dust Storm Warning Advisory and Assessment System (SDS-WAS) to help different countries to combat their sand and dust storm host spots and mitigate the impacts. The WMO and the U.N. Environment also use this model in their policy studies and recommendations.

\section{Conclusion}

This study showed neonatal mortality in Burkina Faso was strongly associated with birth month exposure to dust events. More research needs to be done to understand the extent of the early-life health impacts of exposure to the harsh Saharan SDS better. Also, other West African countries need to be studied separately. More detailed information on mothers' behavior during SDS and indoor air pollution needs to be collected as well. Moreover, the quality of air pollution data needs to be improved by installing monitoring stations-the majority of West African countries do not have air pollution monitors that report to global databases (Brauer et al. 2016). If future studies confirm and expand the recent findings, international coordination is needed to protect the West African populations against the negative health impact of Saharan storms. Currently, such coordination is minimal, while the WMO includes West Africa among the regions that will experience drier weather conditions in the next decade. In the lack of international efforts to address the Saharan storms, domestic health policy should focus on decreasing the long-term effects of the storms on children's health. 


\section{Acknowledgments}

The PM10 concentrations in Senegal, Mali and Niger are provided by the "Service National d'Observation" INDAAF (International Network to study Deposition and Atmospheric composition in AFrica; https://indaaf.obs-mip.fr/) of the French INSUCNRS, the "Observatoire Midi-Pyrénées" (UPS, Toulouse), and the OSU EFLUVE (UPEC, Créteil). The local operators and the technical PIs (A. Féron and C. Gaymoz) are warmly acknowledged for their efforts to maintain the measurement stations and to provide these time series. The COST Action CA16202, supported by COST Association (European Cooperation in Science and Technology) is also acknowledged. Carlos Pérez García-Pando acknowledges the long-term support from the AXA Research Fund, as well as the support received through the Ramón y Cajal programme (grant RYC-2015-18690) of the Spanish Ministry of Economy and Competitiveness, and the European Research Council (ERC) under the European Union's Horizon 2020 research and innovation programme (grant agreement No. 773051).

\section{References}

Al-Hemoud, Ali, Ali Al-Dousari, Ahmad Al-Shatti, Ahmed Al-Khayat, Weam Behbehani, and Mariam Malak. (2018). "Health impact assessment associated with exposure to PM10 and dust storms in Kuwait," Atmosphere, 9:1, 6.

Altindag Duha T., Deokrye Baek, Naci Mocan. (2017). "Chinese Yellow Dust and Korean infant health," Social Science \& Medicine, 1:186, 78-86.

Anderson, Brooke G., and Michelle L. Bell. (2009). "Weather-related mortality: how heat, cold, and heat waves affect mortality in the United States," Epidemiology, 20:2, 205-213.

Bertrand, Marianne, Esther Duflo, and Sendhil Mullainathan. (2004). "How much should we trust differences-in-differences estimates?" Quarterly Journal of Economics, 119:1, 249-275.

Brauer, Michael, Greg Freedman, Joseph Frostad, Aaron van Donkelaar, Randall V. Martin, Frank Dentener, Rita van Dingenen, Kara Estep, Heresh Amini, Joshua S. Apte, Kalpana Balakrishnan, Lars Barregard David Broday, Valery Feigin, Santu Ghosh, Philip K. Hopke, Luke D. Knibbs, Yoshihiro Kokubo, Yang Liu, Stefan Ma, Lidia Morawska, José Luis Texcalac Sangrador, Gavin Shaddick, H. Ross Anderson, Theo Vos, Mohammad H. Forouzanfar, Richard T. Burnett, and Aaron Cohen. (2016). "Ambient air pollution exposure estimation for the global burden of disease 2013," Environmental Science « Technology, 50:1, 79-88.

CIA World Factbook. (2020, February 28). Africa:: Burkina Faso. https://www.cia.gov/library/publications/the-world-factbook/geos/uv.html 
Currie, Janet, Matthew Neidell, and Johannes Schmieder. (2009). "Air pollution and infant health: Lessons from new jersey," Journal of Health Economics, 28:3, 688-703.

Dadvand Payam, Figueras Francesc, Basagaña Xavier, Beelen Rob, Martinez David, Cirach Marta, Marta Cirach, Anna Schembari, Gerard Hoek, Bert Brunekreef, and Mark J. Nieuwenhuijsen. (2013). "Ambient air pollution and preeclampsia: a spatiotemporal analysis," Environmental Health Perspective, 121:11-12, 1365-1371.

Dell, Melissa, Benjamin F. Jones, Benjamin A. Olken. (2012). "Temperature shocks and economic growth: evidence from the last half century," American Economic Journal: Macroeconomics, 4:3, 66-95.

U.S. EPA. (2009). "Integrated Science Assessment (ISA) For Particulate Matter (Final Report, Dec 2009)," U.S. Environmental Protection Agency, Washington, DC, EPA/600/R-08/139F, 2009.

Foreman, Timothy. (2018). "The effect of dust storms on child health in West Africa," CDEP-CGEG Working Paper, No. 47.

Gao, Jin Chun, and Ji Gao Zhang. (2002) "Measurement of electrical charges carried by dust particles [Contact Contamination]." Electrical Contacts, 2002. Proceedings of the Forty-Eighth IEEE Holm Conference On, 191-196.

Garrison, V. H., W. T. Foreman, S. Genualdi, D. W. Griffin, C. A. Kellogg, M. S. Majewski, A. Mohammed, A. Ramsubhag, E. A. Shinn, S. L. Simonich, and G. W. Smith. (2006). "Saharan dust - a carrier of persistent organic pollutants, metals and microbes to the Caribbean?" Revista De Biologia Tropical, 54: 3S, 9-21.

GBD 2016 Risk Factors Collaborators. (2017) “Global, regional, and national comparative risk assessment of 84 behavioural, environmental and occupational, and metabolic risks or clusters of risks, 1990-2016: a systematic analysis for the Global Burden of Disease Study 2016," The Lancet, 390:10100, $1345-1422$.

Gehring Ulrike, Alet H. Wijga, Michael Brauer, Paul Fischer, Johan C. de Jongste, Marjan Kerkhof, Marieke Oldenwening, Henriette A. Smit, and Bert Brunekreef. (2010). "Traffic-related air pollution and the development of asthma and allergies during the first 8 years of life," American Journal of Respiratory and Critical Care Medicine, 181:6, 596-603.

Roland, Gelaro, Will McCarty, Max J. Suárez, Ricardo Todling, Andrea Molod, Lawrence Takacs, Cynthia Randles, Anton Darmenov, Michael G. Bosilovich, Rolf Reichle, Krzysztof Wargan, Lawrence Coy, Richard Cullather, Clara Draper, Santha Akella, Virginie Buchard, Austin Conaty, Arlindo da Silva, Wei Gu, Gi-Kong Kim, Randal Koster, Robert Lucchesi, Dagmar Merkova, Jon Eric Nielsen, Gary Partyka, Steven Pawson, William Putman, Michele Rienecker, Siegfried D. Schubert, Meta Sienkiewicz, and Bin Zhao. (2017). "The Modern-Era Retrospective Analysis for Research and Applications, Version 2 (MERRA-2)," Journal of Climate, 30:13, 5419-5454.

Goldenberg, Robert, Jennifer F. Culhane, Jay D. Iams, and Roberto Romero. (2008). "Epidemiology and causes of preterm birth," The Lancet, 371:9606, 75-84.

Goudie, Andrew S. (2014). "Desert dust and human health disorders," Environment International, 63:C, 101-113. 
Goyal, Nihit, Mahesh Karra, David Canning. (2019). "Early-life exposure to ambient fine particulate air pollution and infant mortality: pooled evidence from 43 low- and middle-income countries," International Journal of Epidemiology, 48:4, 1125-1141.

Heft-Neal, Sam, Jennifer Burney, Eran Bendavid, and Marshal Burke. (2018). "Robust relationship between air quality and infant mortality in Africa," Nature, 559:7713, 254-258.

Heft-Neal, Sam, Jennifer Burney, Eran Bendavid, Kera Voss, and Marshal Burke. (2019). "Air pollution and infant mortality: evidence from Sahara dust," NBER Working Paper, No. 26107.

Ito, Kazuhiko, Samantha F. De Leon, and Morton Lippmann. (2005). "Associations between ozone and daily mortality: analysis and meta-analysis,” Epidemiology, 16:4, 446-457.

Johnston, Fay, Ivan Hanigan, Sarah Henderson, Geoffrey Morgan, and David Bowman. (2011). "Extreme air pollution events from bushfires and dust storms and their association with mortality in Sydney, Australia 1994-2007," Environmental Research, 111:6, 811-816.

Kellogg, Christina A., Dale W. Griffin, Virginia H. Garrison, K. Kealy Peak, Nelson Royall, Raymond R. Smith, and Eugene A. Shinn. (2004). "Characterization of aerosolized bacteria and fungi from desert dust events in Mali, West Africa," Aerobiologia 20:2, 99-110.

Koullali, B., M. A. Oudijk, T. A. J. Nijman, B. W. J. Mol, and E. Pajkrt. (2016). "Risk assessment and management to prevent preterm birth," Seminars in Fetal and Neonatal Medicine, 21:2, 80-88.

Lawn, Joy E., Simon Cousens, and Jelka Zupan. (2005). "4 million neonatal deaths: When? Where? Why?" The Lancet, 365:9462, 891-900.

Lawn, Joy E., Kate Kerber, Christabel Enweronu-Laryea, and Simon Cousens. (2010). "3.6 million neonatal deaths-what is progressing and what is not?" Seminars in Perinatology, 34:6, 371-386.

Li, Xiangyu, Shuqiong Huang, Anqi Jiao, Xuhao Yang, Junfeng Yun, Yuxin Wang, Xiaowei Xue, Yuanyuan Chu, Feifei Liu, Yisi Liu, Meng Ren, Xi Chen, Na Li, Yuanan Lu, Zongfu Mao, Liqiao Tian, and Hao Xiang. (2017). "Association between ambient fine particulate matter and preterm birth or term low birth weight: An updated systematic review and meta-analysis," Environmental Pollution, 227: C, 596-605.

MacIntyre Elaina A., Catherine J. Karr, Mieke Koehoorn, Paul A. Demers, Lillian Tamburic, Cornel Lencar and Michael Brauer. (2011). "Residential air pollution and otitis media during the first two years of life," Epidemiology, 22:1, 81-89.

Matsuura, Kenji, and Cort Willmott. (2007). “Terrestrial air temperature and precipitation: 1900-2010 gridded monthly time series, version 3.01," The University of Delaware. climate.geog.udel.edu/ climate/.

Middleton, Nicholas J. (2017). "Desert dust hazards: a global review," Aeolian Research, 24, 53-63.

Moreira I, C. Linares, F. Follos, G. Sánchez-Martínez, J. M. Vellón, J. Díaz. (2020). "Short-term effects of Saharan dust intrusions and biomass combustion on birth outcomes in Spain," Science of the Total Environment, 701:134755. 
Nenna, Raffaella, Melania Evangelisti, Antonella Frassanito, Carolina Scagnolari, Alessandra Pierangeli, Guido Antonelli, Ambra Nicolai, Serena Arima, Corrado Moretti, Paola Papoff, Maria Pia Villa, and Fabio Midulla. (2017). "Respiratory syncytial virus bronchiolitis, weather conditions and air pollution in an Italian urban area: An observational study," Environmetal Research, 158, 188-193.

Pérez García-Pando, Carlos, Madeleine C. Thomson, Michelle C. Stanton, Peter J. Diggle, Thomas Hopson, Rajul Pandya, Ron L. Miller, Stéphane Hugonnet. (2014a). "Meningitis and climate: from science to practice," Earth Perspectives, 1:14.

Pérez García-Pando, Michelle C. Stanton, Peter J. Diggle, Sylwia Trzaska, Ron L. Miller, Jan P. Perlwitz, José M. Baldasano, Emilio Cuevas, Pietro Ceccato, Pascal Yaka, and Madeleine C. Thomson. (2014b). "Soil Dust Aerosols and Wind as Predictors of Seasonal Meningitis Incidence in Niger," Environmental Health Perspectives, 122:7, 679-686.

Purisch, Stephanie E. and Cynthia Gyamfi-Bannerman. (2017). "Epidemiology of preterm birth," Seminars in Perinatology, 41:7, 387-391.

Ritz, Beate, Fei Yu, Guadalupe Chapa, and Scott Fruin. (2000). "Effect of air pollution on preterm birth among children born in Southern California between 1989 and 1993," Epidemiology, 11:5, 50211.

Schneider, Udo, Andreas Becker, Peter Finger, Anja Meyer-Christoffer, Markus Ziese, and Bruno Rudolf. (2014). "GPCC's new land surface precipitation climatology based on quality-controlled in situ data and its role in quantifying the global water cycle," Theoretical and Applied Climatology, 115:1-2, 15-40.

Soukup, Deborah, Brenda Buck, Dirk Goossens, April Ulery, Brett T. Mclaurin, Dirk Baron, and Yuanxin Teng. (2012). "Arsenic concentrations in dust emissions from wind erosion and off-road vehicles in the Nellis Dunes Recreational Area, Nevada, USA," Aeolian Research, 5, 77-89.

Srám Radim J, Binková Blanka, Jan Dejmek, and Martin Bobak. (2005). "Ambient air pollution and pregnancy outcomes: a review of the literature," Environmental Health Perspectives, 113:4, 375-82.

Tanarhte, M., P. Hadjinicolaou, and J. Lelieveld. (2012). "Intercomparison of temperature and precipitation data sets based on observations in the Mediterranean and the Middle East," Journal of Geophysical Research, 112:D12102.

Thomson, M. C., I. Jeanne, and M. Djingarey. (2009). "Dust and epidemic meningitis in the Sahel: A public health and operational research perspective," IOP Conference Series: Earth and Environmental Science, $7: 1,1-4$.

van Donkelaar, Aaron, Randall V. Martin, Michael Brauer, N. Christina Hsu, Ralph A. Kahn, Robert C. Levy, Alexei Lyapustin, Andrew M. Sayer, and David M. Winker. (2016). "Global estimates of fine particulate matter using a combined geophysical-statistical method with information from satellites, models, and monitors," Environmental Science \& Technology, 50:7, 3762-3772.

WHO (2006). "WHO air quality guidelines for particulate matter, ozone, nitrogen dioxide and sulfur dioxide," Global update 2005, Summary of Risk Assessment, Switzerland. 
WHO (2013). "Review of evidence on health aspects of air pollution," REViHAAP Project, Technical Report; WHO Regional Office for Europe, Denmark.

World Bank (2017). The World Bank World Development Indicators: Burkina Faso. Link: https://data.worldbank.org/country/burkina-faso

World Bank (2020). GDP per capita (constant 2010 US\$) - Senegal, Nigeria, Burkina Faso, Benin, Togo, Niger, Ghana, Cote d'Ivoire. Link: https://data.worldbank.org/indicator/NY.GDP.PCAP.KD?end=2010\&locations=SN-NG-BF-BJTG-NE-GH-CI\&start $=1988$

Xie, Li, Xingcai Li, and Xiaojing Zheng. (2010). "Attenuation of an electromagnetic wave by charged dust particles in a sandstorm," Applied Optics 49:35, 6756-6761.

Xie, Shaodong, Tong Yu, Yuanhang Zhang, Limin Zeng, Li Qi, Xiaoyan Tang X. (2005). "Characteristics of $\mathrm{PM} 10, \mathrm{SO}_{2}, \mathrm{NO}_{x}$ and $\mathrm{O}_{3}$ in ambient air during the dust storm period in Beijing," Science of the Total Environment, 345:1-3, 153-164. 